\title{
Glaucoma Diagnostics for the Clinician 'OPEN YOUR EYES' your Pivotal Role in Improving Glaucoma Care in our High Risk Population
}

SA Lalchan, V Singh

\begin{abstract}
Glaucoma is the world's leading cause of irreversible blindness. Despite this, up to $90 \%$ of patients in the developing world are under diagnosed.

Glaucoma-related blindness in the Caribbean is an eye opener. The earlier age, advanced optic neuropathy, rapid progression coupled with education and cultural barriers all make for a unique challenge.

This review aims to highlight an evidenced-based approach to early diagnosis and the importance of the role of the general practitioner in achieving the paradigm shift towards improved glaucoma care in the Caribbean. The clinician's roles are as follows: firstly, identification of risk factors will aid early referral and diagnosis; secondly, reinforcing the importance of compliance and recognition of side-effects will identify treatment failures; and thirdly, an appreciation of the success of surgical intervention will help support patient's decision.
\end{abstract}

Keywords: Caribbean, general practitioner, glaucoma, glaucoma composites

From: ${ }^{1}$ LILY: The Eye Specialist Limited, Ankavilla Building, \#8 Endeavour Road, Chaguanas. ${ }^{2}$ The Arima Diagnostic Clinic, 25 Hollis Avenue, Eastern Main Road, Arima.

Correspondence: Ms Shelly-Anne Lalchan, The Arima Diagnostic Clinic, 25 Hollis Avenue, Eastern Main Road, Arima. Email: mslalchan@gmail.com 


\section{INTRODUCTION}

Glaucoma is a heterogeneous group of disorders which results in loss of the retinal nerve fibre layer (1). It is a neurodegenerative disease which, to date, the only modifiable risk factor remains the intra-ocular pressure (2). Glaucoma is diagnosed by a trained ophthalmic surgeon as recommended by National Institute for Clinical Excellence [NICE] (3). The outcomes have improved due to early screening, patient education, more effective medical therapies and advanced microsurgical techniques. Nonetheless, glaucoma-related blindness in the Caribbean is an eye opener. The earlier age, advanced optic neuropathy, rapid progression coupled with education and cultural barriers all make for a unique challenge (4).

An estimated 70 million has glaucoma, $10 \%$ of who are blind (5). Despite modern medicine, up to $90 \%$ of patients in developing countries are unaware $(4,6,7,8)$. The reasons are multi-factorial and include patient awareness/education, cultural attitudes and access to professional care. Additionally, policy-makers in developing countries tend to focus on cataracts and refractive errors as these have defined end points (9). There is far less emphasis on glaucoma care as the complexity includes chronicity, high expenditures and skilled longterm professional support. Not surprisingly, it remains the world's leading cause of irreversible blindness (5).

The Caribbean population has one of the highest risks globally $(10,11,12)$. The prevalence in the Barbados Eye Study: in patients of African Descent, >50yrs, 1 in 11 had primary open angle glaucoma (POAG); > 70yrs, was 1 in 6 (13).

Other ethnic-specific evidence shows that in patients of Indian descent the overall risk is lower; but there is a higher risk of developing angle closure glaucoma. Naturally, this is relevant as there are geo-ethnic differences unique to the entire Caribbean. Either way, undoubtedly, the Caribbean population has high risks ${ }^{14}$. The challenges and ergo the solutions are different. 
Notably, many patients also believe that diagnosing glaucoma is simply 'a pressure check' ie the intraocular pressure. Modern diagnosis of early glaucoma includes: tonometry, pachymentry, gonioscopy stereoscopic optic nerve viewing/photography, perimetry and laserassisted optic nerve imaging [Table 1] $(1,3)$.

In the author's opinion, the role of the clinician (general practitioner) is pivotal to moving towards the paradigm shift of evidenced-based improved glaucoma care in the Caribbean. Firstly, identification of risk factors will aid early referral and diagnosis; secondly, reinforcing the importance of compliance and recognition of side-effects will identify treatment failures; and thirdly, an appreciation of the success of surgical intervention will help support patient's decision.

\section{DISCUSSION}

Glaucoma is the world's leading cause of bilateral blindness in patients of African Descent $(7,12,14)$. Despite this, The Barbados Eye Study (BESS) recently showed that even a decade later, $50 \%$ of patients are unaware of having glaucoma. POAG occurs 10yrs earlier, progresses faster and treatment modalities are less efficacious in our ethnicity (12).

The challenges henceforth are as follows:

1 Identification of patients at high risk

2 Early referral to an ophthalmologist/ophthalmic surgeon;

3 Reinforcement of compliance of medications and

4 An appreciation of the success of modern timely surgical intervention.

The general practitioner/clinician is instrumental to all of the above in reducing glaucomarelated blindness. 


\section{Identification of high risk factors and early referral}

All patients with high risk factors for developing glaucoma require a comprehensive glaucoma diagnostic assessment [Table2] $(1,3,5,7)$ and referral to a Consultant ophthalmologist as per NICE guidelines (3). Early treatment can prevent blindness and significantly reduce progression of visual loss; glaucoma related blindness is considered preventable.

Age is a significant risk factor, ie the older one gets the greater the risks (5). Notably, though, glaucoma prevalence was highest in patients of African descent in all age categories. It is well documented that, POAG develops 10yrs earlier in patients of African Descent, therefore patients need to be screened earlier(12,14,17).

In lieu of this, patients need to be assessed as early as $35 \mathrm{yrs}$ of age. Additionally, the diagnosis of advanced glaucomatous optic neuropathy leads to the realization that the disease was present years prior to diagnosis. Of course it slowly progressed with the appearance of symptoms well after $90 \%$ of the nerve is lost ie tunnel vision. This can be prevented with early diagnosis.

Another important risk factor is family history ${ }^{18}$. Patients with a first degree relative of glaucoma have a 10 fold increased risk of developing glaucoma. Young myopic males of African-Descent with a family history of glaucoma have the highest risk (12). There are many patients with these specific demographics. These patients should be seen early by an Ophthalmic Surgeon.

Last, but by no means least, the use of corticosteroids is another factor that can cause elevated intraocular pressures and hence precipitate early glaucoma. These patients need to be informed of ophthalmic complications and be assessed accordingly. Again, the clinician is certainly pivotal at identifying these patients. 


\section{Modern Diagnostics- diagnosing earlier and with precision}

Despite the sheer magnitude of glaucoma, to date there is no specific single examination or test that confirms glaucoma diagnosis, especially in the very early stages. Often, various clinical and diagnostic components have to be considered as pieces of a puzzle.

The author refers to these as the 'glaucoma composites' (Table 1).

Glaucoma composite 1 Tonometry- (IOP).

There are levels of intraocular pressures (IOP) that alert the clinician to an increased risk (Fig 1). But even this can be misleading. Previously, it was believed that an IOP $<21 \mathrm{mmg}$ was normal. There is overwhelming evidence that this is no longer true. In fact, there is evidence that $30 \%$ of POAG occurs at 'normal' intraocular pressures ie glaucoma can and certainly does occur at IOP $<21 \mathrm{mmHg}$. This subset is referred to as normal tension glaucoma (19); but glaucoma specialist simply view this as a spectrum of open angle glaucoma.

This recognition has caused glaucoma specialists to realise that individual optic nerve display varying degrees of pressure sensitivity and clearly, relying on IOP alone is dangerously deceptive.

\section{Glaucoma composite 2 Pachymenty-Central corneal thickness (CCT)}

The Ocular hypertension Treatment Study (OHTS) was a randomised control trial which demonstrated that topical ocular hypotensives can prevent/delay the onset of glaucomatous optic neuropathy by $50 \%{ }^{20}$. Additionally, there was evidence that patients with a CCT of $<555$ um independently had an increased risk (3 fold) of developing glaucoma.

Glaucoma composite 3 Gonioscopy-viewing of the drainage angle.

Gonioscopy allows for diagnosing the various glaucoma subtypes and etiology such as open angle vs primary angle closure vs angle recession (traumatic damage) etc as both the prognosis and treatment strategies are different.

Glaucoma composite 4 Stereoscopic view of the optic nerve 
A dilated stereoscopic view and photographic documentation of the optic nerve are important for both evaluation and objective assessment (20) [Fig 2]. Despite this however, interpretation of the optic nerve for glaucomatous optic neuropathy is also subjective and has been shown to have only $80 \%$ sensitivity and specificity even amongst glaucoma specialist (21). This has prompted the search for more objective imaging modalities.

\section{Glaucoma composite 5 Automated visual field testing/perimetry}

Most clinicians will be familiar with automated visual field testing of perimetry (Fig 2). But, again, up to $50 \%$ of the nerve fibre layer has to be damaged before changes are detected. In the context of early detection and prevention of blindness this is no longer acceptable. There is again a shift towards diagnosing pre-perimetric glaucoma ie before $50 \%$ nerve fibre layer has been irreversible lost.

\section{Glaucoma composite 6 Laser-assisted imaging of the optic nerve}

Optic nerve imaging modalities have been introduced which aims to provide an objective, quantitative analysis and serial monitoring of the optic nerve head parameters and nerve fibre layer (Fig4). These laser scanning modalities include OCT (Optical Coherence Tomography), CSLO (Confocal Scanning Laser Ophthalmoscopy) and HRT [Heidelberg Retinal Tomography- scanning laser polarimetry] $(22,23)$. These modern diagnostics can increase the sensitivity and specificity to $97 \%$ in glaucoma detection (Fig 3). These tests need to be ordered and interpreted within the clinical context in order to correctly diagnose patients.

It is imperative that clinicians appreciate this paradigm shift. It is no longer simply 'a pressure check' by any stretch of the imagination. Blindness from glaucoma is avoidable with early diagnosis and treatment being the key factors. The support of primary care physicians has the potential to reduce the burden of blindness regionally. 


\section{Treatment for glaucoma}

The only known modifiable risk factor for reducing the progressive loss of nerve fiber layer is the intra-ocular pressure. Treatment options for glaucoma include medications, laser therapy and surgery and/or a combination of all three modalities $(1,2,24)$.

\section{Medical therapy}

Medications lower the IOP either by reducing the production or by increasing the rate of outflow of aqueous humour within the eye. Currently, there are five major classes of drugs used for the treatment of glaucoma: (i) prostaglandin analogues (ii) carbonic anhydrase inhibitors (iii) cholinergics (iv) adrenoceptor agonists and (v) beta-adrenoceptor antagonists. The introduction of prostaglandin analogues, has contributed significantly to the successful management of glaucoma. The major benefit was due to its regime of once daily dosing which improved compliance exponentially. The prostaglandin analogues currently are latanoprost $0.005 \%$, travoprost $0.004 \%$ and bimatoprost $0.01 \%$.

The other ocular hypotensives have more frequent dosing. Most of the topicals do have local ocular surface irritation; few, namely, the beta blockers can have systemic side effects and are contraindicated in patients with asthma and bradyarrhythmias

The reduction of the absolute IOP is important hence patients should be encouraged to know the pre-treatment pressures. The ophthalmologist calculates a target IOP that would likely reduce progression. An initial target IOP of $20-50 \%$ reduction serves as a useful guide (1, 24). However, the target IOP has to be continually appraised in the context of the detection of either structural or functional deterioration by the modalities outline above. Quite simply, if the ophthalmologist detects progression, the target pressure should be lower.

Clinicians can aid compliance with medications; help identify reasons for noncompliance; reinforce the irreversibly nature of progressive loss of the optic nerve. Also, the 
recognition of side-effects as this is an important determinant of treatment failure and therefore long-term outcomes.

\section{Laser modalities}

Selective laser trabeculoplasty (SLT) and argon laser trabeculoplasty (ALT) both increase outflow of aqueous humor from the trabecular meshwork. Notably, the efficacy of these interventions wanes with $10 \%$ per year failure rate; additional therapy are usually necessary. In other words, SLT/ALT are temporary measures with patients requiring monitoring for progression and continued medical treatment.

\section{Surgical intervention}

Modern glaucoma filtration procedures are efficacious and demonstrate a good safety profile with low complications for experienced surgeons.

The indications are:

- at presentation with advanced glaucoma or

- uncontrolled IOP or

- progression of glaucoma on maximum tolerated medical treatment (MMT).

- non-compliance

Following treatment failure on maximum medical therapy, surgical intervention is an important option. Modern glaucoma surgery has a success rate of $90 \%$ in the first year; success being qualified by reduction in IOP. There is a failure rate of $5 \%$ per year. Additionally, surgery reduces the diurnal variation in IOP, a know factor in the contribution to glaucoma progression. 
Surgical options include: trabeculectomy augmented with mitomycin-C and glaucoma drainage device (Ahmed/Baerveldt tubes). Increasing, evidence has shown that glaucoma drainage device has better long-term efficacy in controlling the IOP, though complication rates can be higher.

MIGS (Minimally invasive glaucoma surgery) is an emerging field that has some short-term benefit. These devices are however, quite expensive, and may not have long-term costeffectiveness in developing countries.

\section{CONCLUSION}

Primary open angle glaucoma is the world's leading cause of bilateral irreversible blindness. Patients in the Caribbean are amongst the highest risk for developing glaucoma. Prompt referral to an ophthalmologist will aid early diagnosis and effective treatment.

Modern diagnostics need to filter down to developing countries; the role of the clinician can be a pivotal turning point in the management of glaucoma care. These efforts include emphasising the need for early screening; encouraging compliance in medical therapy and adopting an evidenced-based approach to the efficacy of surgical intervention. 


\section{REFERENCES}

1. Cohen LP, Pasquale LR.Clinical Characteristics and Current Treatment of Glaucoma. Cold Spring Harb Perspect Med. 2014 Jun 2; 4(6).

2. The AGIS Investigators. The Advanced Glaucoma Intervention Study (AGIS), 7: the relationship between control of intraocular pressure and visual field deterioration. Am J Ophthalmol. 2000; 130(4):429-440.

3. National Institue for Care and Excellence. Glaucoma: diagnosis and management NICE guidelines [CG85] [Published date April 2009; cited 16 Jan 2016] Available from: http://www.nice.org.uk/guidance/cg85/chapter/Key-priorities-forimplementation

4. Hennis A, Wu SY, Nemesure B, Honkanen R, Leske MC; Barbados Eye Studies Group. Awareness of incident open-angle glaucoma in a population study: the Barbados Eye Studies. Ophthalmology. 2007 Oct; 114 (10):1816-21.

5. Quigley HA, Broman AT. The number of people with glaucoma worldwide in 2010 and 2020. Br J Ophthalmol. 2006; 90(3):262-267.

6. Leite MT, Sakata LM, Medeiros FA. Managing glaucoma in developing countries. Arq Bras Oftalmol. 2011; 74 (2):83-84.

7. Rotchford AP, Kirwan JF, MullerMA, Johnson GJ, Roux P. Temba glaucoma study: a population-based cross-sectional survey in urban South Africa. Ophthalmology. 2003; 110(2):376-382.

8. Sathyamangalam RV, Paul PG, George R et al.Determinants of glaucoma awareness and knowledge in urban Chennai. Indian J Ophthalmol. 2009; 57 (5):355-360.

9. Piergiuseppe Morone, Eva Camacho Cuena, Ivo Kocur, Nicholas Banatvala. Investing in eye health: securing the support of decision-makers. World Health Organization 
2012. [cited 18 Jan 2016] Available from: http://www.who.int/blindness/Politicalanalysis.pdf

10. Hyman L, Wu SY, Connell AM, Schachat A, Nemesure B, Hennis A, Leske MC. Prevalence and causes of visual impairment in The Barbados Eye Study. Ophthalmology. 2001 Oct; 108 (10):1751-6.

11. Mason RP, Kosoko O, Wilson MR, et al: National survey of the prevalence and risk factors of glaucoma in St. Lucia, West Indies. Part I. Prevalence findings. Ophthalmology 96:1363-8, 1989.

12. Racette L, Wilson MR, Zangwill LM, Weinreb RN, Sample PA. Primary open-angle glaucoma in blacks: a review. Surv Ophthalmol. 2003 May-Jun; 48 (3):295-313.

13. Leske MC, Connell AM, Wu SY, Nemesure B, Li X, Schachat A, Hennis A. Incidence of open-angle glaucoma: the Barbados Eye Studies. The Barbados Eye Studies Group. Arch Ophthalmol. 2001 Jan; 119 (1):89-95.

14. Leske MC, Connell AM, Wu SY, et al: Risk factors for open angle glaucoma. The Barbados Eye Study. Arch Ophthalmol 113:918-24, 1995.

15. Goldberg I, Clement CI, Chiang TH, Walt JG, Lee LJ, Graham S, Healey PR. Assessing quality of life in patients with glaucoma using the Glaucoma Quality of Life-15 (GQL-15) questionnaire. J Glaucoma. 2009 Jan; 18(1):6-12.

16. Skalicky S, Goldberg I. Depression and quality of life in patients with glaucoma: a cross-sectional analysis using the Geriatric Depression Scale-15, assessment of function related to vision, and the Glaucoma Quality of Life-15. J Glaucoma. 2008 Oct-Nov; 17(7):546-51.

17. Leske MC, Wu SY, Honkanen R, Nemesure B, Schachat A, Hyman L, Hennis A; Barbados Eye Studies Group. Nine-year incidence of open-angle glaucoma in the Barbados Eye Studies.Ophthalmology. 2007 Jun; 114 (6):1058-64. 
18. Tielsch JM, Katz J, Sommer A, Quigley HA, Javitt JC. Family history and risk of primary open angle glaucoma. The Baltimore Eye Survey. Arch Ophthalmol. 1994 Jan; 112(1):69-73.

19. Mudumbai RC. Clinical update on normal tension glaucoma. Semin Ophthalmol. 2013 May; 28(3):173-9.

20. Gordon MO, Beiser JA, Brandt JD, Heuer DK, Higginbotham EJ, Johnson CA, Keltner JL, Miller JP, Parrish RK 2nd, Wilson MR, Kass MA. The Ocular Hypertension Treatment Study: baseline factors that predict the onset of primary open-angle glaucoma. Arch Ophthalmol. 2002 Jun; 120(6):714-20

20. Zangalli C, Gupta SR, Spaeth GL. The disc as the basis of treatment for glaucoma. Saudi J Ophthalmol. 2011 Oct; 25(4):381-7.

21. Reus NJ, Lemij HG, Garway-Heath DF, Airaksinen PJ, Anton A, Bron AM, Faschinger C, Holló G, Iester M, Jonas JB, Mistlberger A, Topouzis F, Zeyen TG. Clinical assessment of stereoscopic optic disc photographs for glaucoma: the European Optic Disc Assessment Trial. Ophthalmology. 2010 Apr; 117(4):71723.

22. Medeiros FA, Zangwill LM, Bowd C,Weinreb RN. Comparison of the GDx VCC scanning laser polarimeter, HRT II confocal scanning laser ophthalmoscope, and stratus OCT optical coherence tomograph for the detection of glaucoma. Arch Ophthalmol. 2004; 122(6):827-837.

23. Wang YX, O'Leary N, Strouthidis NG, White ET, Ho TA, Garway-Heath DF. Comparison of neuroretinal rim area measurements made by the Heidelberg Retina Tomograph I and the Heidelberg Retina Tomograph II. J Glaucoma. 2013 Oct-Nov 22(8):652-8.

24. American Academy of Ophthalmology Preferred Practice Patterns Committee GP. 
Lalchan and Singh

Preferred practice pattern: primary open-angle glaucoma. In: Ophthalmology. Chicago, Illinois: American Academy of Ophthalmology: 2010. 
Table 1: Modern diagnostic assessment recommended for early diagnosis of glaucoma. The 'Glaucoma Composites'.

\section{Comprehensive Diagnostic Glaucoma Assessment}

Clinical History

Slit lamp examination and anterior segment assessment

Tonometry (measurement of the intraocular pressure)

Gonioscopy (prismatic viewing of the drainage angle)

Pachymetry (measurement of the central corneal thickness)

Dilated fundoscopy for stereoscopic viewing/documentation of the optic nerve

Automated visual field testing

Laser assisted optic nerve imaging(OCT/HRT/GDX)

OCT-optical coherence tomography; HRT- Heidelberg Retinal tomography (Confocal

Scanning Laser Ophthalmoscopy); GDX- Glaucoma Scanning Laser polarimetry

Table 2: Identification of risk factors by the clinician that should prompt early referral to an ophthalmic surgeon/ophthalmologists for a comprehensive diagnostic glaucoma assessment.

\section{Risk Factors for POAG}

1 Age: over $35 y$ rs

2 Ethnicity African Descent

3 Family history of glaucoma

4 Myopia

5 Previous ocular trauma

6 Use of corticosteroids (topical/oral) 


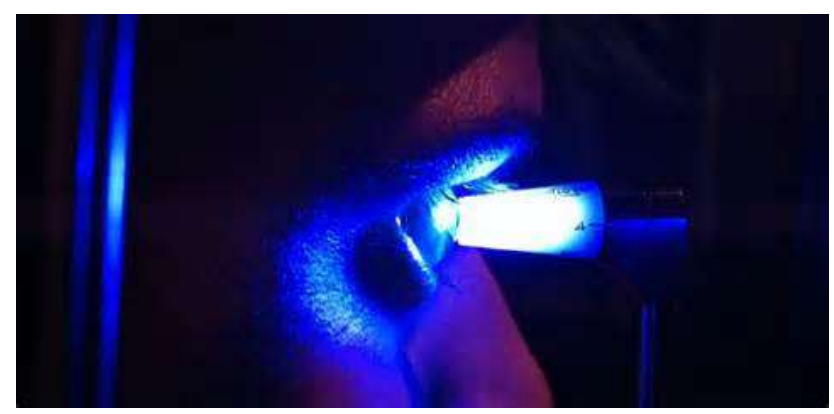

Fig. 1: Goldmann applanation tonometry.

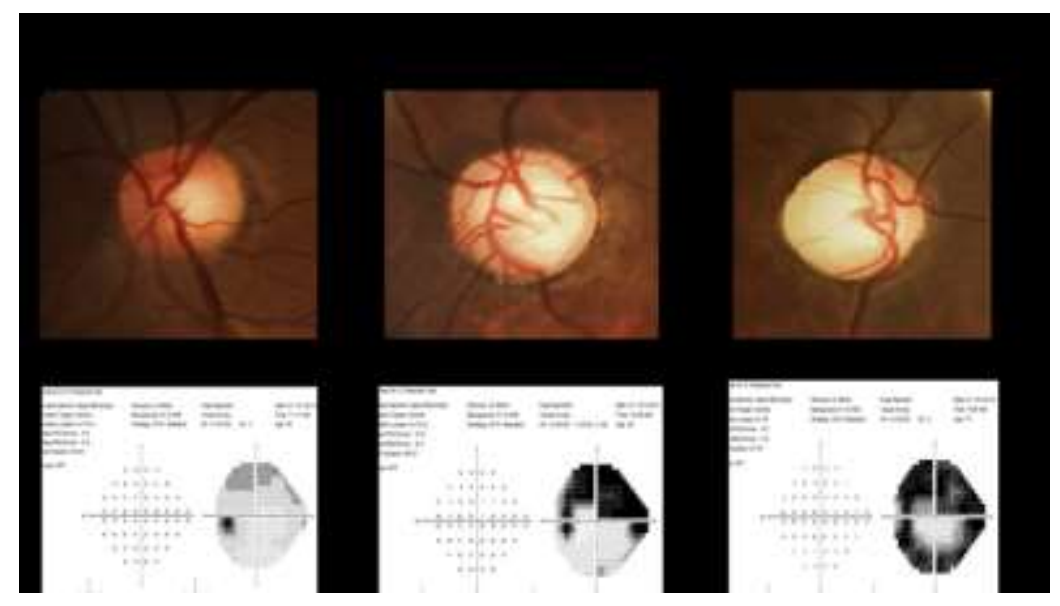

Fig. 2: Optic nerve examination and visual field testing.

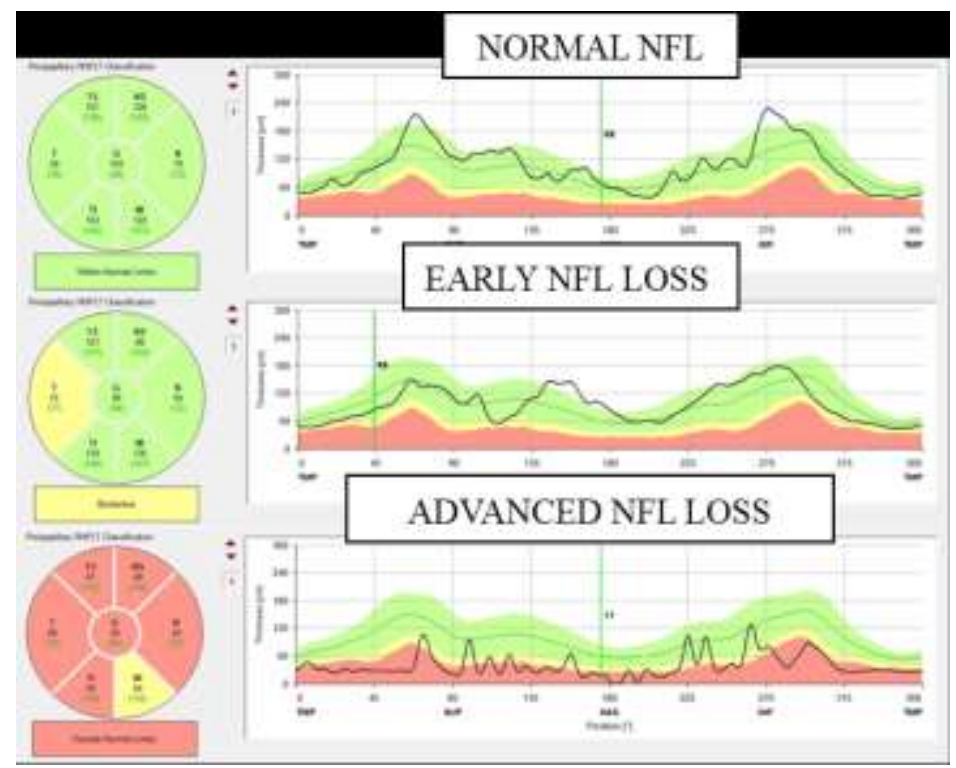

Fig 3: Spectral Domain OCT (Optical Coherence Tomograpy) imaging: nerve fibre layer analysis 